Radiologe $2020 \cdot 60: 737-746$

https://doi.org/10.1007/s00117-020-00685-3

Online publiziert: 29. April 2020

(C) Der/die Autor(en) 2020

Andreas G. Schreyer $\cdot$ L. M. Dendl ${ }^{1,2} \cdot$ G. Antoch ${ }^{3} \cdot$ G. Layer ${ }^{4} \cdot$ L. Beyer ${ }^{5} \cdot$ S. Schleder ${ }^{6}$

'Institut für diagnostische und Interventionelle Radiologie, Klinikum Brandenburg - Medizinische Hochschule Brandenburg, Brandenburg an der Havel, Deutschland

${ }^{2}$ Radiologie, Johanniter Krankenhaus Treuenbrietzen, Treuenbrietzen, Deutschland

${ }^{3}$ Institut für Diagnostische und Interventionelle Radiologie, Universitätsklinikum Düsseldorf, Düsseldorf, Deutschland

${ }^{4}$ Zentralinstitut für Diagnostische und Interventionelle Radiologie, Klinikum Ludwigshafen, Ludwigshafen, Deutschland

${ }^{5}$ Institut für Röntgendiagnostik, Universitätsklinikum Regensburg, Regensburg, Deutschland

${ }^{6}$ Klinik für Diagnostische und Interventionelle Radiologie, Klinikum St. Elisabeth Straubing, Straubing, Deutschland

\title{
Interdisziplinäre
}

\section{Tumorkonferenzen in der radiologischen Routine}

\section{Statuserhebung basierend auf einer Online- Befragung in Deutschland}

Interdisziplinäre Tumorboards sind eine in den letzten Jahren kontinuierlich zunehmende Form regelmäßig stattfindender Konferenzen in der klinischen Routine. Dabei wird durch die Diskussion unter medizinischen Experten verschiedener Fachrichtungen versucht, einen für den einzelnen Patienten optimierten Behandlungsplan durch individuelle Fallbesprechung und Diskussion zu erarbeiten $[1,2]$. Neben dem mittlerweile in verschiedenen Studien nachgewiesenen Nutzen für Patienten haben Boards auch einen interdisziplinären Fortbildungscharakter für die teilnehmenden Ärztinnen und Ärzte [3-8].

In Deutschland hat durch die systematische Pflege und Bearbeitung nationaler Leitlinien und den daraus konsekutiv entstandenen Organ- bzw. Tumorzentren die in diesen Leitlinien und Zertifizierungsvorschriften geforderte Zahl entsprechender Tumorboards überproportional zugenommen [9]. In der Regel wird neben den jeweils direkt betroffenen patientenführenden klinischen Kollegen aus den chirurgischen und konservativen Fächern auch die Anwesenheit eines Pathologen und Radiologen als primär diagnostischen Partnern eingefordert. Bei den meisten Tumorboards sind Radiologen nicht nur als diagnostische, sondern auch als therapeutischer Behandlungspartner im Rahmen der Interventionsradiologie nötig [10].

Obwohl die Anwesenheit eines spezialisierten radiologischen Facharztes in nahezu allen interdisziplinären onkologischen Leitlinien gefordert wird, gehören Radiologen gegenwärtig nach den ASV-Richtlinien des G-BA (Ambulante Spezialärztliche Versorgung - Richtlinie des Gemeinsamen Bundesausschuss) nicht zum „Kernteam" der Tumorboards. Dies verhindert derzeit eine direkte Abrechenbarkeit der erbrachten Leistungen [11].

Durch die massive Zunahme von Tumorboards mit gleichzeitiger Fallzunahme innerhalb der Boards nimmt die Arbeitsbelastung für radiologische Fachärzte in Kliniken signifikant zu. Diese zunehmende Arbeitsbelastung durch sorgfältige Vorbereitung und Präsentation der Fälle wird bisher weder in aktuellen Abrechnungssystemen noch durch eine Kompensation im Stellenschlüssel für die Radiologie adäquat berücksichtigt. Auch die Präsentation und Neubeurteilung extern erstellter
Aufnahmen, sogenannter „Fremdaufnahmen", wird aktuell wirtschaftlich und personell nicht abgebildet.

Geleitet von der Überzeugung, dass die „sprechende Radiologie“ und Tumorboards einer optimalen Patientenversorgung zu Gute kommen, war es das Ziel dieser Untersuchung, die beschriebenen Aufwendungen zu objektivieren und zu analysieren. Dazu führten wir unter den radiologischen Abteilungen der Universitätskliniken sowie ausgewählter großer Kliniken, die ebenfalls entscheidend bei Organ- und Tumorzentren in der Thera-

\footnotetext{
Kernaussagen

- Interdisziplinäre Tumorkonferenzen dienen der optimierten patientenorientierten Therapiefestlegung.

- Die Teilnahme eines radiologischen Facharztes in nahezu allen Boards ist medizinisch unumgänglich.

- Bisher existiert keine systematische Evaluation der Beanspruchung der Radiologie durch Boards.

- Zwischen $50 \%$ und $75 \%$ einer vollen radiologischen Facharztstelle sind für interdisziplinäre Tumorboards nötig.

- Hieraus resultiert ein hoher personeller Aufwand der klinischen Radiologie trotz Ressourcenknappheit.
} 
pie vertreten sind, eine Online-Umfrage durch. Basierend auf dieser Datensammlung wollten wir eine Standortbestimmung der Radiologie bei Tumorboards in Deutschland versuchen. Die ermittelten Zahlen sollten auch Grundlage für eine erweiterte Personalplanung in der Radiologie für die nahe Zukunft liefern.

\section{Material und Methoden}

Die Datenerhebung basierte auf einem Fragebogen für eine Online-Umfrage unter Verwendung des SurveyMonkey® Web Tools (SurveyMonkey Inc., San Mateo, CA, USA, www.surveymonkey.com) in einer professionellen Ausbaustufe, die über die Deutsche Röntgengesellschaft (DRG) zur Verfügung gestellt wurde [12].

Die Einladung zur Teilnahme an der Umfrage wurde in einem direkten, persönlichen E-Mail-Kontakt an alle Lehrstuhlinhaber in der Radiologie oder deren Vertreter der 33 deutschen Universitätskliniken verschickt. Weiterhin wurden Einladungen deutschlandweit nach Zufallsprinzip an 50 Chefärzte für Radiologie oder deren Vertreter an Krankenhäusern der Schwerpunkt-, oder Maximalversorgung (im Folgenden „Schwerpunktkliniken “ genannt) in einem direkten, persönlichen E-Mail-Kontakt verschickt. Bei Nichtteilnahme erfolgte jeweils eine einmalige Erinnerung, ebenso in einem direkten, persönlichen E-MailKontakt 3 Monate nach Erstkontakt.

Insgesamt wurden in der Umfrage 30 Fragen online gestellt. Zunächst wurden Informationen zu Daten des Teilnehmers und der Klinik abgefragt. Die Fragen 6 bis 17 waren auf die interdisziplinären Tumorkonferenzen bezogen und ergaben Informationen zu den verschiedenen Fachbereichen, in denen diese durchgeführt wurden, zu Dauer und Fallzahl der jeweiligen interdisziplinären Tumorkonferenzen und zur tatsächlich aufgewendeten Vorbereitungszeit. Ferner wurde abgefragt, ob die interdisziplinären Tumorkonferenzen im Rahmen zertifizierter Zentren stattfanden und welche klinischen Fachrichtungen sich regelhaft beteiligten. Weiterhin wurden Informationen zur Einschätzung der Häufigkeit externer Bildbefunde und zum diesbezüglichen diagnostischen Mehraufwand gewonnen. Abschließend wurde eruiert, welchen Ausbildungsstatus (Assistenzarzt, Facharzt, Oberarzt, Chefarzt) die teilnehmenden Radiologen bei Tumorkonferenzen hatten und wie hoch die Einflussnahme der Radiologie auf den Therapieentscheid im Board subjektiv eingeschätzt wurde.

Die so gewonnenen Daten wurden mit deskriptiver Statistik (Microsoft Excel for Mac, Version 16.20, Microsoft Corp., Redmond, WA, USA) ausgewertet.

Zur Berechnung der durchschnittlichen Zahl interdisziplinärer Tumorkonferenzen, zur Ermittlung der durchschnittlichen Dauer und Vorbereitungszeiten sowie zur Ermittlung der mittleren Fallzahlen wurden jeweils die Mittelwerte der zur Auswahl stehenden Antwortmöglichkeiten als Berechnungsgrundlage genutzt.

Zusätzlich wurde ein Webtool programmiert, in dem basierend auf den Ergebnissen dieser Online-Umfrage getrennt nach Universitätskliniken und Schwerpunktkliniken die jeweils individuelle zeitliche Belastung durch Vorbereitung und Durchführung der Boards nach Eingabe der jeweiligen Anzahl der durchgeführten Boards errechnet werden kann (https://lit-scrubland-86478. herokuapp.com). Die Implementierung der Webseite basiert auf der komponentenbasierten JavaScript Bibliothek React und dem Bootstrap Framework [13].

\section{Ergebnisse}

\section{Rücklaufquote}

Bei der Online-Umfrage nahmen bei den Universitätskliniken 26 von 33 radiologischen Instituten $(79 \%)$ und bei den Schwerpunktkliniken 28 von 50 Instituten $(56 \%)$ teil.

\section{Bettenzahl und Stellenschlüssel}

Die durchschnittliche Bettenzahl der teilnehmenden Universitätskliniken und Schwerpunktkliniken betrug 1362 bzw. 963. Der gemittelte Stellenschlüssel (Chefarzt - Oberarzt - Assistenz- oder Facharzt) der radiologischen Hauptab- teilungen wurde an Universitätskliniken mit 1-10-27 und an Schwerpunktkliniken mit 1-6-13 angegeben.

\section{Dauer, Vorbereitungszeit und \\ Fallzahl interdisziplinärer \\ Tumorkonferenzen}

Die durchschnittliche Durchführungszeit (• Abb. 1), Vorbereitungszeit (- Abb.2) und die jeweiligen besprochenen Fallzahlen (•Abb. 3) schwankten zwischen den unterschiedlichen interdisziplinären Tumorkonferenzen deutlich. Die höchsten Fallzahlen fielen insgesamt in den interdisziplinären senologischen Fallbesprechungen, der Neuroonkologie und den abdominellen Tumorentitäten an. An den Universitätskliniken wurden dementsprechend die höchsten Fallzahlen in den Boards für Mammaund gynäkologische Tumoren (20 Fälle), Hirntumoren (18 Fälle) und allgemeine gastrointestinale Tumoren inkl. Pankreas (17 Fälle) verzeichnet, während an den Schwerpunktkliniken die höchsten Fallzahlen mit durchschnittlich 11, 10 und 10 Fällen bei den Boards für Mamma- und gynäkologische Tumoren, Lungentumoren und allgemeine gastrointestinale Tumoren inkl. Pankreas vorlagen.

Im Vergleich der Universitätskliniken $\mathrm{zu}$ den Schwerpunktkliniken spiegelte sich der höhere Versorgungsgrad und die Bettenzahl der mehr belasteten Universitätskliniken. Entsprechend dauerte eine interdisziplinäre Tumorkonferenz an einer Universitätsklinik $49 \mathrm{~min}$, verglichen mit $38 \mathrm{~min}$ an einer Schwerpunktklinik. Die durchschnittliche Vorbereitungszeit für den durchführenden Radiologen betrug an Universitätskliniken $53 \mathrm{~min}$, während der Zeitaufwand an Schwerpunktkliniken etwa $30 \mathrm{~min}$ betrug. Die mittlere Fallzahl wurde mit 13,5 Fällen in der universitären Versorgung angegeben, wohingegen die gemittelte Fallzahl an Schwerpunktkliniken mit etwa 6,5 Fällen genannt wurde (• Abb. 1, 2 und 3).

Interdisziplinäre Tumorkonferenzen wurden regelhaft - je nach klinischem Schwerpunktfach - sowohl an den Universitätskliniken als auch an den Schwerpunktkliniken für alle Tumorentitäten mehrfach pro Woche abgehalten, wo- 
Radiologe 2020 - 60:737-746 https://doi.org/10.1007/s00117-020-00685-3

(c) Der/die Autor(en) 2020

A. G. Schreyer · L. M. DendI · G. Antoch · G. Layer · L. Beyer · S. Schleder

\section{Interdisziplinäre Tumorkonferenzen in der radiologischen Routine. Statuserhebung basierend auf einer Online-Befragung in Deutschland}

\section{Zusammenfassung}

Ziel. Interdisziplinäre Tumorkonferenzen (Boards) sind regelmäßig stattfindende Konferenzen, bei denen unter medizinischen Experten verschiedener Fachrichtungen versucht wird, einen für den Patienten optimierten Behandlungsplan zu erstellen. Die Anwesenheit eines radiologischen Facharztes ist in nahezu allen relevanten Boards medizinisch unumgänglich. Um die aktuelle Beanspruchung der Radiologie durch derartige Boards systematisch zu evaluieren und auch um aktuelle Zahlen zur möglichen zukünftigen Personalplanung zu liefern, haben wir eine aktuelle Standortbestimmung in der Radiologie in Deutschland durchgeführt. Material und Methoden. Es erfolgte eine Online-Umfrage. Zur Teilnahme aufgefordert waren alle 33 Lehrstuhlinhaber in der Radiologie sowie nach dem Zufallsprinzip weitere 50 ausgewählte Chefärzte für Radiologie an Krankenhäusern der Schwerpunkt-, oder Maximalversorgung (im Folgenden "Schwerpunktkliniken" genannt).

Ergebnisse. Es nahmen 26 von 33 radiologischen Instituten (79\%) der Universitätskliniken und 28 von 50 Instituten (56\%) der Schwerpunktkliniken teil. Die Gesamtzahl der durchgeführten Tumorboards beträgt im Durchschnitt 3,3 pro Tag bzw. 16,7 pro Woche an Universitätskliniken und 2,6 pro Tag bzw. 13 pro Woche an Schwerpunktkliniken. Es ergibt sich ein durchschnittlicher zeitlicher Aufwand unter Berücksichtigung der Vorbereitungs- und Durchführungszeit sowie der Durchführungshäufigkeiten von $33,1 \mathrm{~h} /$ Woche an Universitätskliniken und 18,2 h/Woche an Schwerpunktkliniken. Dies entspricht bei einer 42-Stunden-Woche an Universitätskliniken $78,8 \%$ und bei einer 40 -Stunden-Woche an Schwerpunktkliniken 45,5\% einer Facharzt- stelle, die für interdisziplinäre Tumorboards notwendig sind.

Schlussfolgerung. Die "sprechende“ Radiologie mit ihren interdisziplinären Tumorboards stellt ein klinisches Selbstverständnis unter aktiver Teilnahme und Moderation durch die Radiologie dar, welches die Patientenversorgung evidenzbasiert verbessert. Dennoch muss bei der in der Medizin vorherrschenden Ressourcenknappheit für künftige Diskussionen bezüglich einer personellen Kompensation die hier erhobene Datengrundlage bzgl. des Personalaufwands der klinischen Radiologie für die Teilnahme an Tumorboards bedacht werden.

Schlüsselwörter Wirtschaftlichkeit - Entscheidungshilfe . Kosteneffizienz - Gesundheitspolitik . Tumorboard

\section{Interdisciplinary tumor boards in the radiological routine. Current situation based on an online survey in Germany}

\section{Abstract}

Purpose. Interdisciplinary tumor boards are periodical conferences, where optimal individual therapy plans are developed among medical experts with different specializations. The presence of a board-certified radiologist is medically indispensable in almost all relevant boards. In order to systematically evaluate the current workload for radiologists caused by these boards, we evaluated the current situation within German radiology to obtain numbers for future personnel planning. Materials and methods. We performed an online survey. We invited all 33 German university chairmen and 50 randomly selected head physicians of radiology at level 3 hospitals to participate.
Results. We had a participation rate of $79 \%$ (26/33) at university hospitals and $56 \%(28 / 50)$ at of level 3 non-university hospitals. The average total number of tumor boards was $3.3 /$ day or $16.7 /$ week at university hospitals and $2.6 /$ day or 13 /week at level 3 nonuniversity hospitals. We calculated an average time considering preparation and execution as well as the average number of boards of $33.1 \mathrm{~h} /$ week for university hospitals and $18.2 \mathrm{~h} /$ week for level 3 hospitals. This results in a $78.8 \%$ workload for a board-certified radiologist at a university hospital (regular weekly work time $42 \mathrm{~h}$ ) and $45.5 \%$ work load for level 3 hospitals (regular weekly work time $40 \mathrm{~h})$.
Conclusion. "Speaking radiology" as in interdisciplinary tumor boards represents a fundamental matter of course in radiology. The active participation in boards accomplished by radiologists improves evidence-based patient care. However, given the prevailing scarcity of resources in medicine, the data collected here regarding personnel costs for clinical radiology for participation in tumor boards must be taken into account in future discussions on personnel compensation.

Keywords

Economics - Decision analysis - Costeffectiveness - Health policy and practice . Tumor board bei die mittlere Häufigkeit gemittelt über alle Boards an Universitätskliniken mit 1,4 Besprechungen je Tumorentität gegenüber 1,2 Konferenzen an den Schwerpunktkliniken etwas höher lag (• Abb. 4).

Betrachtet man nun den gesamten Arbeitsaufwand für die Radiologie zur Teilnahme an den interdisziplinären Tumorkonferenzen unter Berücksichtigung der mehrmaligen wöchentlichen Durchführung einzelner Boards mit der jeweiligen Durchführungsdauer und den entsprechenden Vorbereitungszeiten, so ergab sich ein durchschnittlicher Zeitaufwand pro interdisziplinärer Tumorkonferenz von 142 min pro Woche an Universitätskliniken und von $78 \mathrm{~min}$ pro Woche an Schwerpunktkliniken (• Abb. 5).
Damit ergab sich für radiologische Abteilungen unter Berücksichtigung der Vorbereitungs- und Durchführungszeit ein durchschnittlicher zeitlicher Aufwand von $18,2 \mathrm{~h}$ pro Woche an Schwerpunktkliniken und von $33,1 \mathrm{~h}$ pro Woche an Universitätskliniken.

Dies entspricht, unter der Annahme einer tariflichen 40-Stunden-Woche an Schwerpunktkliniken $45,5 \%$ einer 


\section{Originalien}

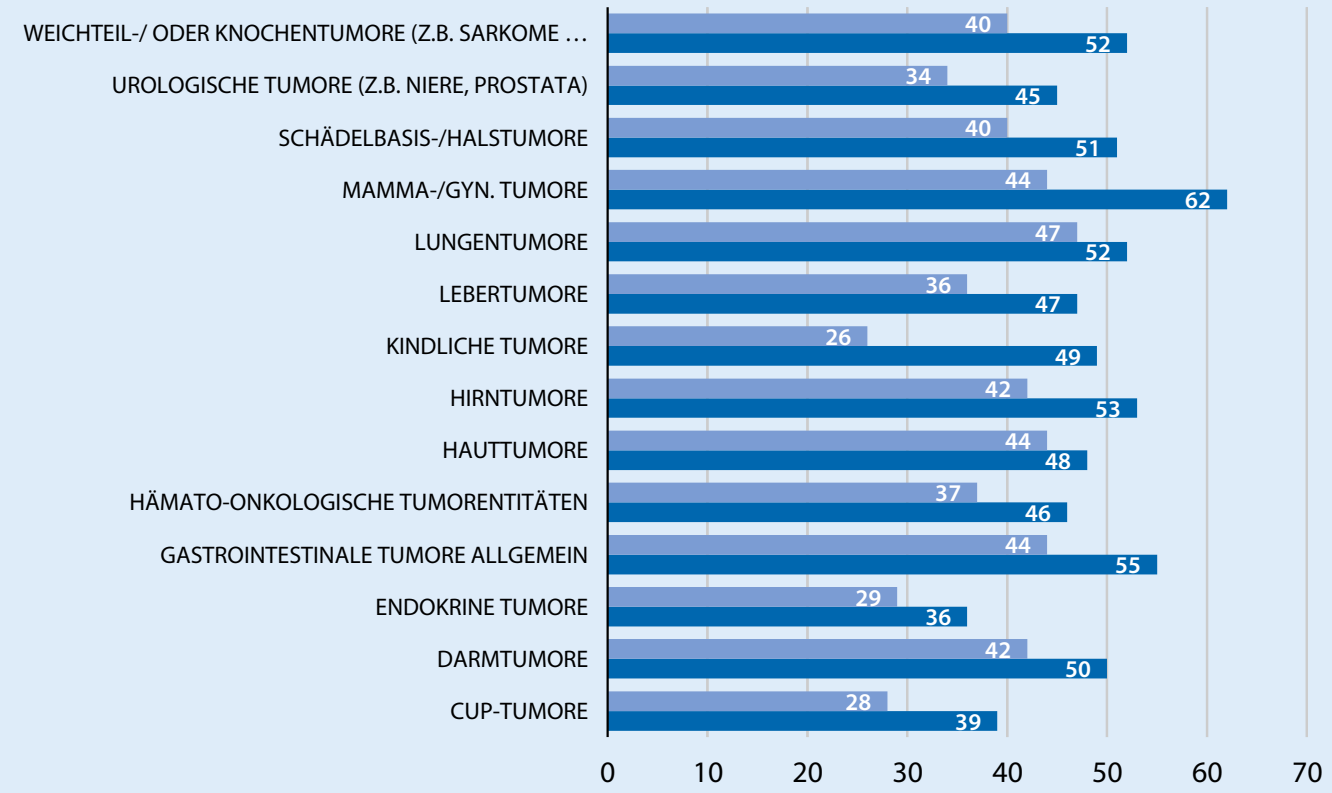

Kliniken der Regel-, Schwerpunkt-, und Maximalversorgung — Universitätskliniken
Abb. $1 \triangleleft$ Durchschnittliche Dauer (in Minuten) einzelner interdisziplinärer Tumorkonferenzen an Universitätskliniken (dunkelblau) und an Schwerpunktkliniken (hellblau)

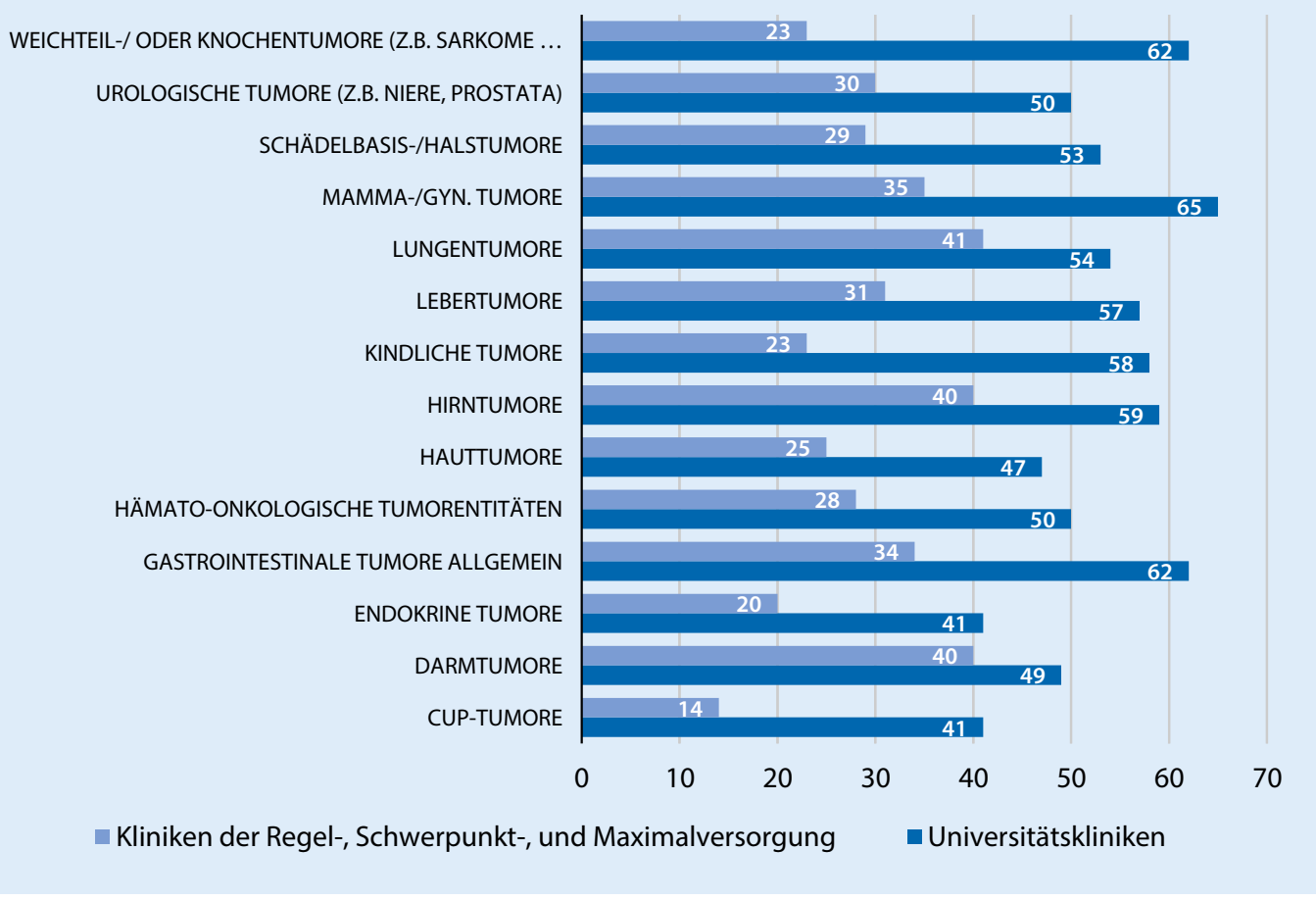

Abb. $2<$ Durchschnittliche Vorbereitungszeit (in Minuten) einzelner interdisziplinärer Tumorkonferenzen an Universitätskliniken (dunkelblau) und an Schwerpunktkliniken (hellblau)
Facharztstelle und unter der Annahme einer tariflichen 42-Stunden-Woche an Universitätskliniken 78,8\% einer Facharztstelle. Eine individualisierte Einschätzung des Arbeitsaufwandes durch interdisziplinäre Tumorboards ist basierend auf unseren Auswertungsdaten auf einem Netzrechner unter https://lit- scrubland-86478.herokuapp.com/ möglich.

\section{Extern erstellte Aufnahmen in Tumorboards}

An den Schwerpunktkliniken und Universitätskliniken antworteten jeweils
$65 \%$, dass im Rahmen von interdisziplinären Tumorboards subjektiv mehr als $25 \%$ extern erhobene radiologische Bilddaten demonstriert wurden (• Tab. 1). Bei den Schwerpunktkliniken gehen $27 \%$, bei den Universitätskliniken sogar $53 \%$ der Befragten davon aus, dass mehr als die Hälfte des Vorbereitungsaufwan- 

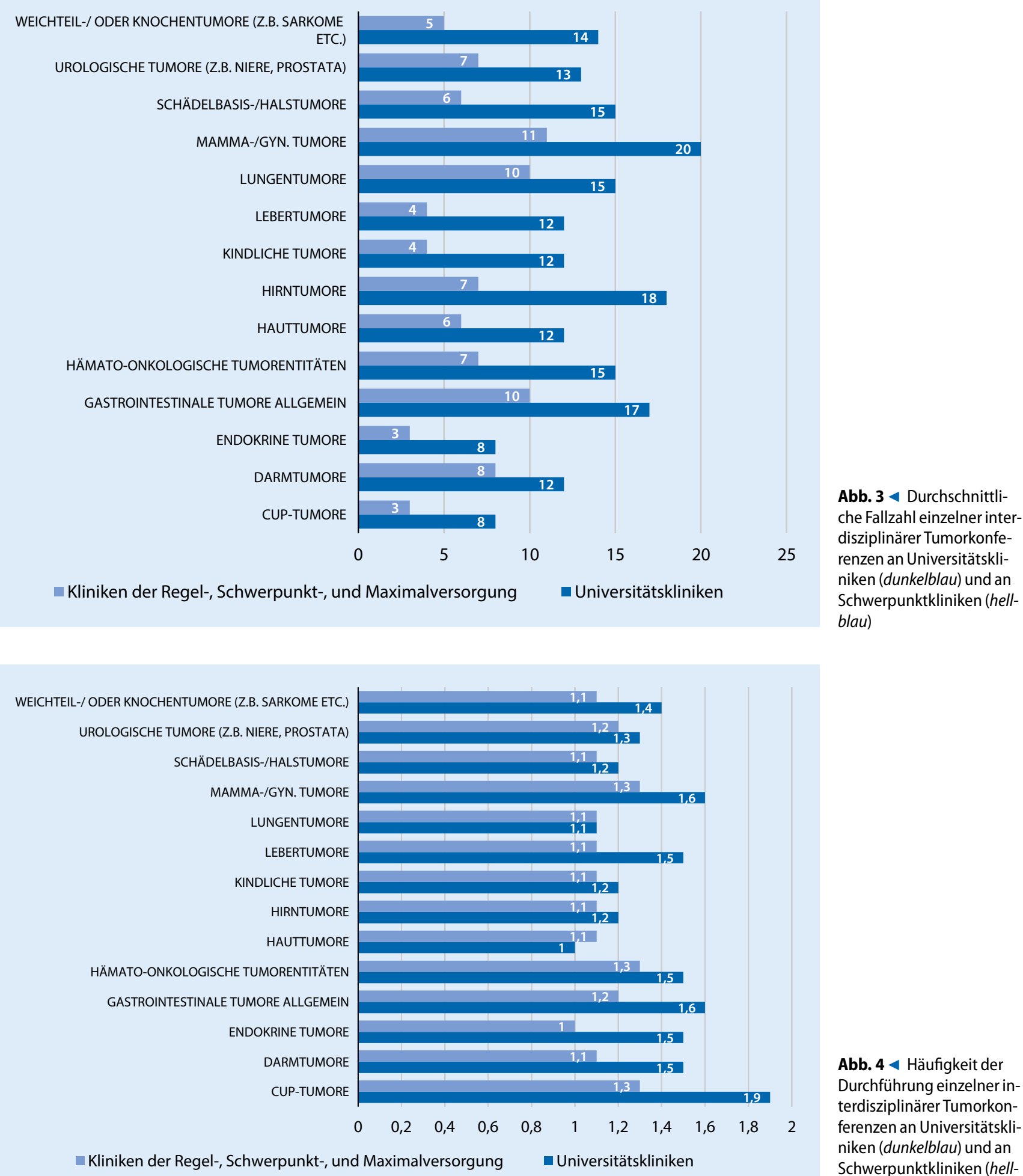

Abb. $4<$ Häufigkeit der Durchführung einzelner interdisziplinärer Tumorkonferenzen an Universitätskliniken (dunkelblau) und an Schwerpunktkliniken (hellblau)

des durch extern erhobenes Bildmaterial entstanden war (• Tab. 1). Dabei lag nach Einschätzung der befragten Radiologen nur den wenigsten externen Bilddaten auch die zugehörigen schriftlichen Befunde für das Tumorboard bei: $39 \%$ der
Kollegen an den Schwerpunktkliniken und $61 \%$ an den Universitätskliniken gaben an, dass in $10 \%$ oder weniger der Fälle der primäre Befundbericht zur Verfügung stand (•Tab. 1).
An den Schwerpunktkliniken wurden bei $72 \%$, an den Universitätskliniken bei $81 \%$ der Teilnehmer extern akquirierte Untersuchungen ohne den primären Befundbericht in den Boards demonstriert, während Schwerpunktkliniken in 


\section{Originalien}

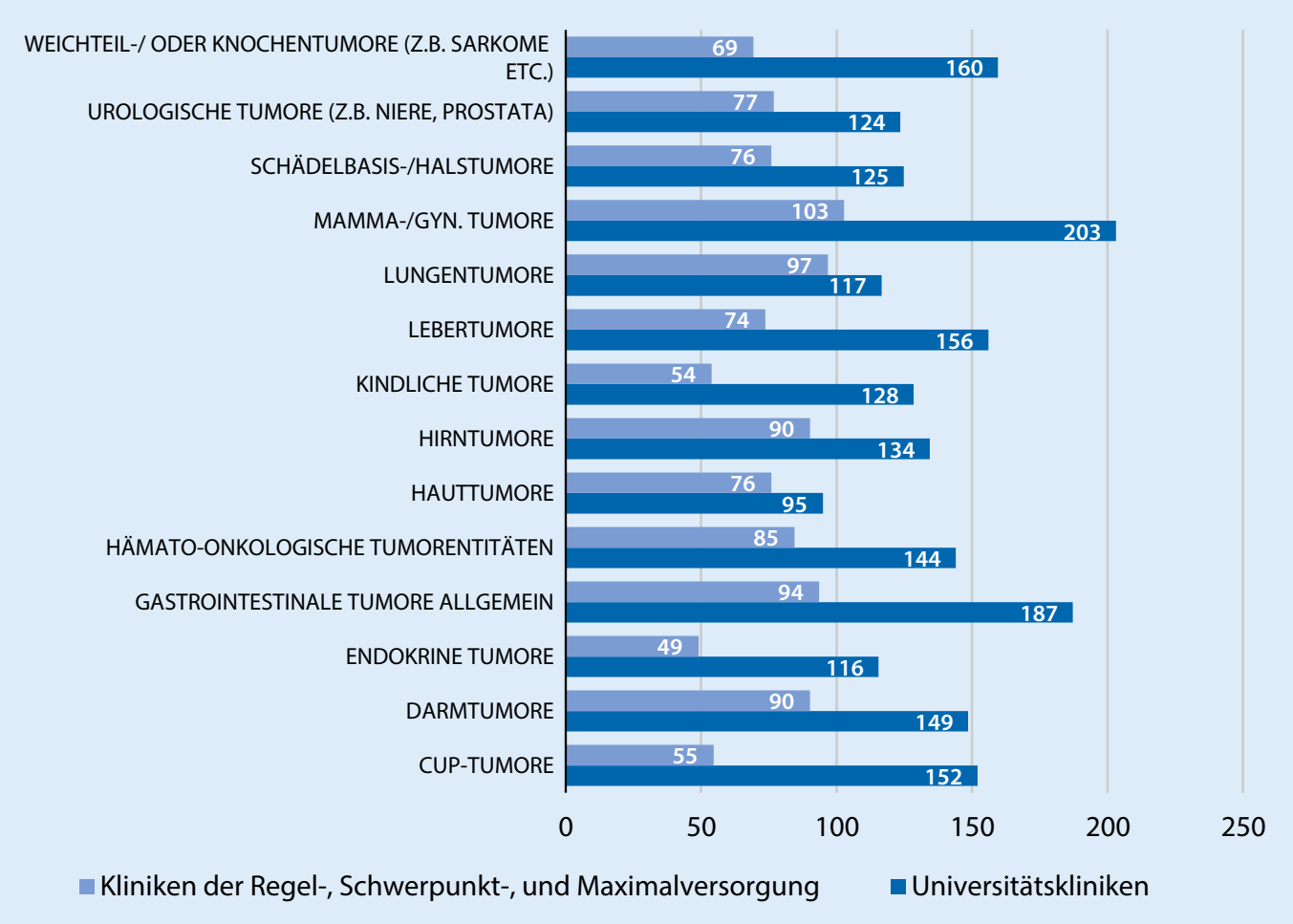

Abb. $5<$ Durchschnittliche Arbeitszeit pro einzelner interdisziplinärer Tumorkonferenzen pro Woche (in Minuten) an Universitätskliniken (dunkelblau) und an Schwerpunktkliniken (hellblau) unter Berücksichtigung der Häufigkeit, der Dauer der Durchführung und der Vorbereitungszeit

$28 \%$ und Universitätskliniken in $19 \%$ das Vorliegen des primären Befundberichtes für eine Bild-Demonstration in interdisziplinären Tumorboards forderten.

\section{Einschätzung des radiologischen Einflusses auf Therapie- entscheidungen im Tumorboard}

Bei der Frage nach dem subjektiv geschätzten Einfluss der bildgebenden Diagnostik auf Therapieentscheide im Rahmen interdisziplinärer Tumorkonferenzen entfielen 0 bzw. $4 \%$ auf die Antwortmöglichkeit 1-10\%, $17 \%$ bzw. $46 \%$ auf die Antwortoption 10-25\% und 26\% bzw. $23 \%$ auf $25-50 \%$. $27 \%$ der Teilnehmer aus Schwerpunktkliniken und sogar $55 \%$ derer aus Universitätskliniken waren der Meinung, dass der Radiologe den Therapieentscheid in über $50 \%$ der vorgestellten Fälle beeinflusste.

\section{Diskussion}

Mit einer Gesamtzahl von insgesamt 54 Teilnehmern bei einer Rücklaufrate von $79 \%$ der deutschen Universitätskliniken und 28 von 50 (60\%) Teilnehmern der angeschriebenen großen Kliniken der Schwerpunkt- und Maximalversorgung darf die vorgelegte Auswertung zur Arbeitsbelastung durch interdisziplinäre Tumorkonferenzen in der radiologischen Routine als repräsentative Datenerhebung in deutschen Großkliniken betrachtet werden. Bisher gibt es keine genauen belastbaren Zahlen durch vergleichbare Erhebungen. Lediglich eine Analyse aus dem Jahr 2012 aus den USA lässt vermuten, dass damals ein radiologischer Facharzt etwa $14 \%$ der Arbeitszeit für Röntgenbesprechungen und weitere $10 \%$ mit informellen Falldemonstrationen verbrachte [14]. Dies bezieht sich jedoch auf alle klinischen Befunddemonstrationen und ist daher mit der hier vorliegenden Fragestellung nur sehr eingeschränkt vergleichbar.

Interdisziplinäre Tumorboards ermöglichen durch die fachübergreifende Diskussion von medizinischen Experten eine Individualisierung und Optimierung der Behandlungspfade von onkologischen Patienten. In nahezu allen aktualisierten onkologischen Leitlinien wird eine Durchführung von Tumorboards empfohlen. Alle Zertifizierungsrichtlinien der Deutschen Krebsgesellschaft fordern regelmäßige Konferenzen als
Qualitätsmerkmal bei der Zertifizierung von Organzentren. Es resultierte in den letzten Jahren eine massive Zunahme organ- bzw. tumorspezifischer Tumorboards vor allem an Universitätskliniken und großen, nichtuniversitären Kliniken der Schwerpunkt- und Maximalversorgung.

Dem Nutzen von interdisziplinären Tumorboards stehen aber organisatorische, personelle und damit wirtschaftliche Herausforderungen der klinisch beteiligten Behandlungspartner und hier insbesondere der Radiologie gegenüber. Die hohe Personalbindung klinisch tätiger Radiologen durch Vorbereitung, Teilnahme und Moderation der Tumorboards wurde bislang nicht systematisch ausgewertet. In einer Analyse zur Wertigkeit der klinischen Relevanz von Röntgenbesprechungen in der Inneren Medizin wurde dabei eine durchschnittliche Vorbereitungsdauer pro Fall von 5,1 min mit einer Spanne zwischen $1 \mathrm{~min}$ und $20 \mathrm{~min}$ angegeben [15].

Alleine die Anzahl von wöchentlich durchschnittlich 14,5 Boards an Universitätskliniken und von 10,9 Boards an Schwerpunktkliniken zeigt die diesbezüglich hohe Arbeitsbelastung in der Ra- 
Tab. 1 Geschätzter prozentualer Anteil extern erhobenen Bildmaterials, geschätzter Mehraufwand durch extern erhobenes Bildmaterial, geschätzte Häufigkeit des Vorhandenseins eines schriftlichen Befundes bei extern erhobenem Bildmaterial jeweils an Universitätskliniken und an Schwerpunktkliniken (Anzahl/Prozent) in Tumorboards

\begin{tabular}{|c|c|c|c|c|c|c|}
\hline & \multicolumn{2}{|c|}{ Extern erhobenes Bildmaterial } & \multicolumn{2}{|c|}{$\begin{array}{l}\text { Mehraufwand durch externes Bild- } \\
\text { material }\end{array}$} & \multicolumn{2}{|c|}{$\begin{array}{l}\text { Vorhandensein eines schriftlichen Befundes bei } \\
\text { extern erhobenem Bildmaterial }\end{array}$} \\
\hline & $\begin{array}{l}\text { Universitäts- } \\
\text { kliniken (n) \% }\end{array}$ & $\begin{array}{l}\text { Schwerpunkt- } \\
\text { kliniken }(n) \%\end{array}$ & $\begin{array}{l}\text { Universitäts- } \\
\text { kliniken }(n) \%\end{array}$ & $\begin{array}{l}\text { Schwerpunkt- } \\
\text { kliniken }(n) \%\end{array}$ & $\begin{array}{l}\text { Universitätskliniken } \\
\text { (n) \% }\end{array}$ & $\begin{array}{l}\text { Schwerpunktkliniken } \\
\text { (n) \% }\end{array}$ \\
\hline $1-10 \%$ & (1) 4 & (0) 0 & (1) 4 & (1) 4 & (14) 61 & (10) 39 \\
\hline $10-25 \%$ & (2) 9 & (9) 35 & (0) 0 & (5) 19 & (5) 22 & (4) 15 \\
\hline $25-50 \%$ & (15) 65 & (12) 46 & (10) 44 & (13) 50 & (3) 13 & (5) 19 \\
\hline $50-75 \%$ & (4) 17 & (5) 19 & (10) 44 & (6) 23 & (1) 4 & (4) 15 \\
\hline $\begin{array}{l}\text { Mehrals } \\
75 \%\end{array}$ & (1) 4 & (0) 0 & (2) 9 & (1) 4 & (0) 0 & (3) 12 \\
\hline
\end{tabular}

diologie. Die Universitätskliniken haben im Vergleich zu großen Scherpunktkliniken durchschnittlich neben der Anzahl an Boards eine größere Fallzahl sowie damit eine längere Vorbereitungszeit und Durchführungszeit. Für ein Universitätsklinikum ergibt sich im Durchschnitt ein wöchentlicher personeller Gesamtaufwand für einen radiologischen Facharzt zur Vorbereitung und Teilnahme an Boards von $33,1 \mathrm{~h}$ pro Woche und für große Schwerpunktkliniken ein Aufwand von 18,2 h. Durchschnittlich wird somit die Stelle eines Facharztes am Universitätsklinikum $\mathrm{zu} 78,8 \%$ und am Schwerpunktklinikum zu 33,1\% durch interdisziplinäre Boards eingenommen. Bemerkenswert ist die Einschätzung von $53 \%$ der universitären Teilnehmer und $27 \%$ der nichtuniversitären Befragten, dass mehr als die Hälfte des Vorbereitungsaufwandes von Tumorboards mit der Beurteilung externer Bilddaten verbracht werden muss. Gleichzeitig berichten $61 \%$ der universitären und 39\% der nichtuniversitären Befragten, dass der radiologische Erstbefund für die Vorbereitung der Tumorboards nicht oder nur sehr eingeschränkt zur Verfügung steht.

Juristisch besteht nach den strahlenschutzrechtlichen Grundsätzen prinzipiell eine Einheit von Bild und Primärbefundung [16]. Die neue Beurteilung eines bereits extern befundeten radiologischen Bildes stellt somit keine eigene Leistung dar [11]. Im Gegenteil können ernsthafte juristische Probleme entstehen, wenn die radiologischen Beurteilungen differieren, da der konsiliarisch tätige Experte trotz fehlender Entlohnung die volle juristische Verantwortung für seine getroffene Einschätzung trägt [16].

Diese Bildung einer Expertenmeinung wird bislang auch personell oder budgetär nicht berücksichtigt. Nach dem Institut für das Entgeltsystem im Krankenhaus (InEK) abrechnungsfähig - ein Institut, welches die Krankenhäuser und Krankenkassen sowie deren Verbände bei der gesetzlich vorgeschriebenen Einführung und kontinuierlichen Weiterentwicklung des G-DRG-Systems zur Abrechnung von stationären Krankenhausleistungen unterstützt - sind nur selbst erbrachte diagnostische Leistungen, Zweit- und Expertenmeinung dagegen nicht, obwohl der ärztliche Arbeitsaufwand vergleichbar ist und lediglich die technischen Leistungen entfallen. Das bedeutet vor allem für große Kliniken einen hohen Verlust an potenziellen Einnahmen. Für die einzelne radiologische Abteilung heißt es aber vor allem, dass diese zur Qualität des Krankenhauses in hohem Maß beitragende Leistung quasi bestraft wird. Sie ist kalkulatorisch durch das InEK nicht abgebildet und wird daher auch nicht im Budget berücksichtigt. Je mehr Konferenzen unterstützt werden und je häufiger eine qualifizierte Zweitmeinung geliefert wird, desto defizitärer arbeitet eine Abteilung. Krankenhausmanager betrachten i. d. R. in erster Linie die Kostenstruktur der Radiologie und nicht den nur schwer ermittelbaren Mehrwert dieser Disziplin. Allenfalls fließt neben Budgeteinhaltung und Erlösen noch die Termintreue und die Befundlaufzeit in Bewertungsmaßstäbe ein, die enorm wichtige Beratungsleistung der klinischen Partner dagegen selten bis nie.

Die Einschätzung des Einflusses der radiologischen Beteiligung am Ergebnis des Tumorboards ist objektiv nur schwer $\mathrm{zu}$ fassen. Der Benefit in Diagnose und Therapie konnte allerdings an kleineren Studien über die sprechende Radiologie gezeigt werden [15]. Bei Tumorboards lassen sich in der Literatur dagegen keine validen Zahlen finden, die den Einfluss der Radiologie quantifizieren. Lediglich in der oben erwähnten Arbeit zur Analyse einer radiologischen Konferenz in der Inneren Medizin konnte eine Änderung diagnostischer und therapeutischer Pfade von $37 \%$ gezeigt werden [15]. Eine Translation der Ergebnisse auf interdisziplinäre Tumorboards ist nur eingeschränkt möglich. Hier schätzten $81 \%$ der universitären Teilnehmer ihren Einfluss auf Therapieentscheidungen bei über $25 \%$ der Fälle ein sowie $60 \%$ der Teilnehmer aus Schwerpunktkliniken.

Die Herausnahme der Radiologie aus den Kernteams der ambulanten spezialärztlichen Versorgung (ASV), in der bereits aktuell die Tumorerkrankungen des Mammakarzinoms und der gastrointestinalen Tumoren behandelt werden, verhindert zudem eine direkte Abrechnung radiologischer Leistungen in diesem System, obwohl auch hier fast immer mindestens ein Radiologe als diagnostischer und interventioneller Experte an den interdisziplinären Konferenzen teilnimmt. Fachlich ist es selbstverständlich nicht vorstellbar, dass eine Patientin mit Mammakarzinom ohne einen Experten für bildgebende Diagnostik erfolgreich behandelt wer- 
den kann. Trotzdem wird dies vom Gesetzgeber bzw. Gemeinsamen Bundesausschuss systematisch negiert. Ohne auch betriebswirtschaftliche Beteiligung wird es aber weder niedergelassenen senologischen Diagnostikern noch radiologischen Klinikabteilungen dauerhaft möglich sein, hochwertige Leistungen $\mathrm{zu}$ erbringen und zum Therapieerfolg und der individualisierten Therapie des häufigsten Tumors der Frau beizutragen. Dann wäre das Ziel der ASV, „Patienten mit schweren Krankheitsverläufen durch eine enge Verzahnung von Spezialisten verschiedener Fachdisziplinen besser zu versorgen“, konterkariert.

Die Deutsche Krebsgesellschaft(DKG) schreibt für die von ihr zertifizierten Organzentren eine mindestens einmal wöchentlich stattfindende Tumorkonferenz vor. Zur Teilnahme auf Facharztniveau verpflichtet sind die Fachrichtungen diagnostisches, operatives und ggf. organspezifisch-medikamentöses Fachgebiet, Radioonkologie, Hämatologie und Onkologie, Radiologie sowie Pathologie (Nationales Zertifizierungsprogramm Krebs der Deutschen Krebsgesellschaft vom 02.11.2017). Hieraus wird klar ersichtlich: Ohne das Fachgebiet der Radiologie finden keine Tumorboards statt, bei denen Bildgebung erforderlich ist, eine Entscheidung auf Boden des schriftlichen Befundes alleine ist nicht legitim. Viele Kliniken sind gezwungen, aufgrund der hohen Fallzahl (• Abb. 3) den vorgeschriebenen Turnus für $\mathrm{Tu}$ morkonferenzen zu unterschreiten und diese mehrfach wöchentlich abzuhalten (• Abb. 4), was zu einer zusätzlichen Belastung führt.

\section{Schlussfolgerung}

Zusammenfassend muss betont werden, dass sowohl die sprechende Radiologie mit ihren Röntgenkonferenzen als auch die Zunahme interdisziplinärer Tumorboards unter aktiver Teilnahme und Moderation durch die Radiologie ein klinisches Selbstverständnis darstellt, welches die Patientenversorgung evidenzbasiert verbessert. Dennoch muss bei der in der Medizin üblichen Ressourcenknappheit für künftige Diskussionen bezüglich einer personellen Kompensation eine solide Datengrundlage des Personalaufwands der klinischen Radiologie für die Teilnahme an Tumorboards vorliegen.

Der durchschnittliche wöchentliche zeitliche Aufwand alleine für interdisziplinäre Tumorboards beträgt dabei in unserer Umfrage zwischen 18 und $33 \mathrm{~h}$ je nach Art der Klinik, wobei dadurch $50-75 \%$ einer vollen radiologischen Facharztstelle für die Vorbereitung und Durchführung der Tumorboards nötig sind.

\section{Klinische Relevanz}

- Die aktive Teilnahme an interdisziplinären Tumorkonferenzen durch die Radiologie ist sowohl an Universitätskliniken als auch an Schwerpunktkliniken sehr zeit- und personalintensiv.

- Ein großer Anteil des Arbeitsaufwandes zur Vorbereitung von Tumorboards liegt vermutlich in der erneuten Aufarbeitung extern erstellter Aufnahmen, deren Primärbefundung häufig nicht zur Verfügung steht.

- Der Beitrag der Radiologie an Tumorboards zu Änderungen der Diagnose- und Therapiepfade dürfte in Zusammenschau mit der Literatur und den subjektiven Angaben der Befragten bei über $30 \%$ liegen.

\section{Korrespondenzadresse}

Prof. Dr. Andreas G. Schreyer

Institut für diagnostische und Interventionelle Radiologie, Klinikum Brandenburg -

Medizinische Hochschule Brandenburg

Hochstraße 29, 14770 Brandenburg an der

Havel, Deutschland

andreas.schreyer@mhb-fontane.de

Funding. Open Access funding provided by Projekt DEAL.

\section{Einhaltung ethischer Richtlinien}

Interessenkonflikt. A.G. Schreyer, L.M. Dendl, G. Antoch, G. Layer, L. Beyer und S. Schleder geben an, dass kein Interessenkonflikt besteht.

Für diesen Beitrag wurden von den Autoren keine Studien an Menschen oder Tieren durchgeführt. Für die aufgeführten Studien gelten die jeweils dort angegebenen ethischen Richtlinien.
Open Access. Dieser Artikel wird unter der Creative Commons Namensnennung 4.0 International Lizenz veröffentlicht, welche die Nutzung, Vervielfältigung Bearbeitung, Verbreitung und Wiedergabe in jeglichem Medium und Format erlaubt, sofern Sie den/die ursprünglichen Autor(en) und die Quelle ordnungsgemäß nennen, einen Link zur Creative Commons Lizenz beifügen und angeben, ob Änderungen vorgenommen wurden.

Die in diesem Artikel enthaltenen Bilder und sonstiges Drittmaterial unterliegen ebenfalls der genannten Creative Commons Lizenz, sofern sich aus der Abbildungslegende nichts anderes ergibt. Sofern das betreffende Material nicht unter der genannten Creative Commons Lizenz steht und die betreffende Handlung nicht nach gesetzlichen Vorschriften erlaubt ist, ist für die oben aufgeführten Weiterverwendungen des Materials die Einwilligung des jeweiligen Rechteinhabers einzuholen.

Weitere Details zur Lizenz entnehmen Sie bitte der Lizenzinformation auf http://creativecommons.org/ licenses/by/4.0/deed.de.

\section{Literatur}

1. Gross GE (1987) The role of the tumor board in a community hospital. CA Cancer JClin 37(2):88-92

2. Haier J, Bergmann KO (2013) Medicolegal aspects of tumor boards. Chirurg 84(3):225-230

3. Jalil R, Akhter W, Lamb BW, Taylor C, Harris J, Green JS et al (2014) Validation of team performance assessment of multidisciplinary tumor boards. JUrol 192(3):891-898

4. Lamb BW, Green JS, Benn J, Brown KF, Vincent CA, Sevdalis N (2013) Improving decision making in multidisciplinary tumor boards: prospective longitudinal evaluation of a multicomponent intervention for 1,421 patients. J Am Coll Surg 217(3):412-420

5. Mattes MD, Gerbo R, Dattola RM (2017) Tumor board shadowing for medical students as a means of early exposure to multidisciplinary oncology education. J Am Coll Radiol 14(2):253-255

6. Petty JK, Vetto JT (2002) Beyond doughnuts: tumor board recommendations influence patient care. JCancer Educ 17(2):97-100

7. Sarff M, Rogers W, Blanke C, Vetto JT (2008) Evaluation of the tumor board as a continuing medical education (CME) activity: is it useful? JCancer Educ 23(1):51-56

8. Wheless SA, McKinney KA, Zanation AM (2010) A prospective study of the clinical impact of a multidisciplinary head and neck tumor board. Otolaryngol Head Neck Surg 143(5):650-654

9. El Saghir NS, Keating NL, Carlson RW, Khoury KE, Fallowfield L (2014) Tumor boards: optimizing the structure and improving efficiency of multidisciplinary management of patients with cancer worldwide. Am Soc Clin Oncol Educ Book. https:// doi.org/10.1155/2014/875897

10. Greten TF, Malek NP, Schmidt S, Arends J, Bartenstein P, Bechstein W et al (2013) Diagnosis of and therapy for hepatocellular carcinoma. ZGastroenterol 51(11):1269-1326

11. Schreyer AG, Steinhauser RT, Rosenberg B (2018) Externally acquired radiological data for the clinical routine-a review of the reimbursement situation in Germany. Rofo 190(05):427-432

12. Goldberg DT Selina, Maly Tim, Aldrich Will (2015) https://de.surveymonkey.com/ 
13. Wikipedia (2017) Bootstrapping. https://de wikipedia.org/wiki/Bootstrap_(Framework). Zugegriffen:28. Juli 2017

14. MacDonald SL, Cowan IA, Floyd R, Mackintosh S, Graham R, Jenkins E et al (2013) Measuring and managing radiologist workload: application of lean and constraint theories and production planning principles to planning radiology services in a major tertiary hospital. J Med Imaging Radiat Oncol 57(5):544-550

15. Dendl LM, Teufel A, Schleder S, Rennert J, Stroszczynski C, Mueller-Schilling M et al (2017) Analysis of radiological case presentations and their impact on therapy and treatment concepts in internal medicine. Rofo 189(3):239-246

16. Schreyer AG, Rosenberg B, Steinhauser RT (2018) Externally acquired radiological image data and reporting for the clinical routine, conference and boards-legal aspects of the second opinion in Germany. Rofo 190(7):610-615

\section{Engelhardt, Monika, Mertelsmann, Roland, Duyster, Justus (Hrsg.) Das Blaue Buch}

Chemotherapie-Manual Hämatologie und Onkologie

Berlin Heidelberg: Springer-Verlag 2020, 7. Auflage, 815 S., (ISBN: 978-3-66260379-6), Softcover 69,99 EUR

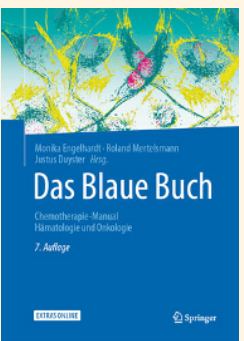

Die Durchführung einer wissenschaftlich fundierten, bestmöglichen Therapieoption sollte das Ziel eines jeden Behandlers in der Versorgung von Tumorpatienten sein.

Der rasante Fortschritt in der Systembehandlung von Krebserkrankungen führt zwangsläufig ständig zu neuen Behandlungsmöglichkeiten, die in der Fülle kaum detailliert zu überblicken sind. Die Gruppe der Clinical Cancer Research (CCR)-Group (vormals GCP-Team) am Universitätsklinikum Freiburg um Engelhardt/Mertelsmann/Duyster hat es sich seit 1994 zur Aufgabe gemacht, die kontinuierliche Aktualisierung, Validierung und Qualitätssicherung von Behandlungsprotokollen zu gewährleisten. Diese "GCPWerkzeuge" sind seit 2007 als „Das Blaue Buch" veröffentlicht und gelten mittlerweile als das Standardwerk eines ChemotherapieManuals. Zentrale Komponenten der standardisierten Chemotherapie-Durchführung sind dabei die Chemotherapie-Protokollblätter als Übersicht mit Angaben über den Therapieablauf und die jeweilige Begleitmedikation. Grundlegend überarbeitet ist nun die 7. Auflage des Manuals erschienen. Enthalten sind $>500$ detailliert ausgearbeitete und in der klinischen Praxis etablierte Behandlungsprotokolle in Tabellenform, wobei neben klassischen Chemotherapie-Protokollen auch Handlungsanweisungen für neue innovative Therapien (z.B. Small molecules, ImmunCheckpointinhibitoren) enthalten sind. Jedes einzelne Protokoll beinhaltet einen kompletten zeitlich dargestellten Verfahrensablauf, angefangen von der Begleitmedikation über die Applikation der einzelnen Chemotherapeutika bis hin zu Warnhinweisen und detaillierten Hilfen für die Verlaufskontrollen. Dabei werden nicht nur orale und intravenöse Systemtherapien dargestellt, sondern auch intrakavitäre Chemotherapien, Mobilisierungschemotherapien zur Sammlung von Stammzellen, autologe und allogene Kon-
ditionierungs-Protokolle sowie supportive Therapieprotokolle. Mittels Internetzugriff sind neben den Therapieprotokollen auch SOPs und klinische Behandlungspfade zugänglich. Als besonders hilfreich und von großer Praxisrelevanz ist die übersichtliche Dosismodifikationstabelle einzelner Substanzen bei Nieren- oder Leberinsuffizienz zu erwähnen, die in der aktuellen Auflage wieder gründlich überarbeitet wurde. Bewährt haben sich seit der letzten Auflage die Behandlungs-Protokolle der kombinierten Radio-/Chemotherapie. Interessant ist ferner die Integration einer in Freiburg entwickelten Planungs- und Verwaltungssoftware, die eine digitale Komplettlösung für eine Behandlungseinrichtung darstellt (ChemoCompile). Zusammenfassend kann festgehalten werden, dass das Blaue Buch mit seinen über 500 Protokollen mittlerweile ein Klassiker eines therapieorientierten Nachschlagewerkes ist und uneingeschränkt das komplette Rüstzeug für die sichere State-of-the-art Behandlung im Alltag eines jeden Hämatologen und Onkologen bietet.

Prof. Dr. Dr. h.c. Uwe Martens, Heilbronn 
Hier steht eine Anzeige.

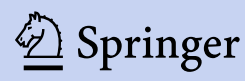

